IBARS ALMONACIL, A. M. \& ESTRELLES PERPIÑÁ, E. -2000- Una nueva localidad de Oenothera speciosa Nutt. para la Península Ibérica. Fl. Montibérica 16: 25-26.

NIETO FELINER, G. -1990- Armeria L. In: S. CASTROVIEJO M. LAINZ, G. LÓPEZ, P. MONSERRAT, F. MUÑOZ GARMENDIA, J. PAIVA \& L. VILLAR Flora Ibérica 2: 642-721.

PAU, C. -1899- La «Oenothera speciosa» en España. 'Actas Soc. Esp. Hist. Nat. 28: 212-213.

POMATA, E. -1882- Catálogo de plantas recolectadas al estado espontáneo en la provincia de Toledo. Anales Soc. Esp. Hist. Nat. 11(2-3): 241-306

ROMERO ZARCO, C. -1984- Revisión taxonómica del género Avenula (Dumort.) Dumort. (Gramineae) en la Península Ibérica e Islas Baleares. Lagascalia 13(1): 39-146.

SANTOS, M. T., M. LADERO \& A. AMOR -1989Vegetación de las intercalaciones básicas de la provincia de Cáceres (Extremadura, España). Stvdia Botanica 11: 9-147.
TERRACCIANO, A. -1905- Revisiones monografica delle Gagea della Flora Spagnola. Bol. Soc. Aragonesa Ci. Nat. 4: 188-253

VALDÉS, B. -1987- Solanaceae In: B. VALDÉS, S. TALAVERA \& E. FERNÁNDEZ GALIANO. Flora de Andalucia Occidental 2:351-362.

VÁZQUEZ, F.M. \& J.A. DEVESA -1988Fragmenta Chorologica Ocidentalia 1966-2000. Anales del Jardín Botánico de Madrid 45(2): 532-534.

Aceptado para su publicación en octubre de 2004

Dirección de los autores. Sección de Producción Forestal. Servicio de Investigación y Desarrollo Tecnológico. Consejería de Agricultura y Medio Ambiente. Junta de Extremadura. Avd. de Portugal s/n 06800 Mérida (Badajoz).

\title{
139. NUEVAS CITAS Y CORRECCIONES A FLORA IBERICA PARA PLANTAS DEL NW DE LA PROVINCIA DE LEÓN
}

\author{
Ruth MARTÍNEZ ARIAS, Ana FERNÁNDEZ \\ RODRÍGUEZ y Marta Eva GARCÍA GONZÁLEZ
}

New records and corrections to Flora iberica to plants of northwest of Leon province.

Palabras clave. Corología, León, España, Flora iberica.

Key words. Chorology, Leon, Spain, Flora iberica.

A raíz de nuevos estudios florísticos en el noroeste de la provincia de León, más en concreto en la cuenca minera de la comarca de El Bierzo, hemos constatado la presencia de una serie de táxones no citados anteriormente para dicha provincia; de igual modo, hemos observado algunas imprecisiones en las áreas de distribución de otros que 
aparecen en los tomos publicados de Castroviejo et al.(1986-2003).

Señalamos con un asterisco (*) aquellas especies que constituyen novedad provincial; con el símbolo (\#), los táxones para los que Flora iberica no indica la sigla provincial, Le. Los que van precedidos por el símbolo $(+)$ indican los señalados, en esta obra, para la provincia con dudas geográficas o taxonómicas -Le?-. Por último, aparecen con un guión (-) aquellos táxones para los que Flora iberica basa su presencia en León en el testimonio de un especialista o en alguna cita fiable, pero no respaldada por pliegos de herbario -(Le)-.

\section{+ Asplenium trichomanes L. subsp. trichomanes}

LEÓN: La Silva (Villagatón) (29TQH243205) 12-V-2002, entre rocas, $880 \mathrm{~m}$, R. Martínez-Arias, LEB 79656.

Citas bibliográficas numerosas distribuidas por toda la provincia, como Cuevas del Sil (29TQH15), Puente García (1988); Valdelugueros (30TUN06), López Pacheco (1988); Santa Lucía de Gordón (30TTN85), Pérez Morales (1988); Sierra de la Cabrera, La Baña, camino del lago (29TPG9182), Nieto Feliner (1985); Molinaferrera (29TQG19), Llamas García (1984); entre otros. En ocasiones puede haber sido confundido este taxon con la subsp. quadrivalens que está presente también en la provincia pero, a diferencia de la anterior, presenta las pinnas superiores insertas oblicuamente al raquis, más o menos distantes y con un tamaño ligeramente menor. Nuestros ejemplares deben ser incluidos, sin duda, en la subespecie trichomanes.

\section{Aster lanceolatus Willd.}

LEÓN: Embalse de Bárcena, Toreno (29TQH044260) 23-VIII-2002, sauceda, $625 \mathrm{~m}$, R. Martínez-Arias, LEB 79193.

Segunda mención de esta especie para la provincia de León, que ya fue citada por vez primera en Villager de Laciana (29TQH15), Puente García (1988). Taxon que en la Cornisa Cantábrica se halla solamente en las provincias de Asturias y León.
Avena barbata Pott ex Link subsp. lusitanica (Tab. Mor.) Romero Zarco

LEÓN: Matachana-Turienzo Castañero (Castropodame) (29TQH099189) 2-VI-2001, prado con cultivo de pinos, $740 \mathrm{~m}$, R. MartínezArias, LEB 78806.

Citada por primera vez por Romero Zarco (1990) en la localidad de Rozuelo (29TQH21). La nuestra representa la segunda mención provincial.

\section{* Cuscuta campestris Yuncker}

LEÓN: Embalse de Bárcena, Santa Marina del Sil (Toreno) (29TQH039257) 6-IX-2001, parásita de Xanthium strumarium subsp. strumarium, $620 \mathrm{~m}$, R. Martínez-Arias, LEB 78736.

Nuestra primera cita provincial es importante porque supone una ampliación muy significativa de su área de distribución hacia los territorios noroccidentales. Se trata de la población situada más al noroeste de España.

\section{Cynoglossum creticum Miller}

LEÓN: Arlanza (Bembibre) (29TQH123282) 9-VI-2001, borde de carretera, 735 m, R. Martínez-Arias, LEB 78425.

Taxon que ya fue citado por Pau (1983), cerca de León capital (30TTN81) y por Paz Canuria et al. (1999) en León y Cea-San Pedro (30TUN364033). Nuestra cita supone una ampliación significativa de la zona de distribución de esta especie, dentro de la provincia, hacia el noroeste.

+ Cytinus hypocistis (L.) L. subsp. hypocistis

LEÓN: Río Primout, carretera minera a Alinos (Toreno) (29TQH043341) 26-V-2001, sobre raíces de Cistus salviifolius, $730 \mathrm{~m}$, R. Martínez-Arias, LEB 78363.

Citada anteriormente por García Glez. et al. (1987) en Destriana (29TQG38) parasitando a Cistus laurifolius y Halimium alyssoides; la nuestra supone la segunda localidad del taxon para la provincia.

\section{Echium asperrimum Lam.}

LEÓN: Boeza (Folgoso de la Ribera) (29TQH214308) 16-VI-2001, escombrera de carbón, 1020 m, R. Martínez-Arias, LEB 78134; 
Valencia de Don Juan 27-VII-1976, Penas, LEB 6495.

Taxon de ambientes ruderalizados que ha sido citado una sola vez para la provincia en Valderas (30TTM96), Penas Merino (1984). Nuestra cita de Boeza supone una ampliación significativa del área de distribución hacia el oeste español, ya que no existen poblaciones más noroccidentales que las encontradas por nosotros.

\section{* Gagea villosa (Bieb.) Duby}

LEÓN: Manzanal del Puerto (Villagatón) (29TQH284183) 13-IV-2002, restos de brezal, 1190 m, R. Martínez-Arias, LEB 78712.

Segunda mención para la provincia de esta especie que se desarrolla en ribazos y pastos secos, que fue citada en León, en el Valle de Lechada, por debajo de Peña Prieta (30TUN56), por Laínz (1964).

Nuestra cita representa la población española más noroccidental.

\section{*Galinsoga ciliata (Rafin) S.F. Blake}

LEÓN: Bárcena de la Abadía (Fabero) (29TPH944410) 29-VIII-2001, cultivos hortícolas, $795 \mathrm{~m}$, R. Martínez-Arias, LEB 78634; Villanueva de Carrizo (30TTN61), sitio húmedo al lado de cultivos, C. Pérez Morales, LEB 49367.

Esta planta característica de bordes de carretera, cunetas, jardines y huertas, presenta las escamas del receptáculo enteras, a diferencia de G. parviflora (común en la provincia), además de que las escamas del vilano son aristadas y los pedúnculos tienen pelos glandulares patentes de más de $0,5 \mathrm{~mm}$.

La nuestra representa además la primera cita para la comunidad de Castilla y León de este taxon que aparece muy puntualmente en el territorio español.

\section{Hedera hibernica (G. Kirchn.) Bean}

Siguiendo a Valcárcel et al. (2003), se procedió a la revisión de los materiales del género en LEB y se concluyó que a pesar de que habían sido citados como Hedera helix, pertenecían en realidad al taxon $H$. hibernica. Además, como ya aventuraban dichos autores para la provincia de León, los tricomas de las hojas jóvenes presentan características intermedias entre esta especie y
H. maderensis subsp. iberica. Los tricomas en las hojas eran de dos tipos, ambos sésiles, pero de diferente tamaño y coloración, unos anaranjados menores de $0,5 \mathrm{~mm}$ y con $8-9$ radios y otros blanquecinos, con menor número de radios (7-9), de tamaño ligeramente mayor pero soldados en un mayor porción.

\section{Illecebrum verticillatum $\mathrm{L}$.}

LEÓN: Embalse de Bárcena, Toreno (29TQH039256) 23-VIII-2002, canal seco, R. Martínez Arias, LEB 79204.

Taxon importante por estar presente en la Lista de Flora Amenazada Leonesa, Llamas et al. (2003), que ya fue citada en la localidad de Burbia, bajo el Mustallar (Vega de Espinareda) (29TPH7942) por Silva Pando (1984) y en Tabuyo del Monte (Luyego) (29TQG38) por Llamas García (1984).

\section{- Lathyrus cicera L.}

LEÓN: Matachana-Turienzo Castañero (Castropodame) (29TQH099190) 1-V-2002, borde de carretera, $730 \mathrm{~m}$, R. Martínez-Arias, LEB 78685.

Citado anteriormente en Aviados (30TUN04), prado al borde del robledal, López Pacheco (1988); La Pola de Gordón (30TTN84) y Geras de Gordón (30TTN75), Pérez Morales (1988). Nuestra mención supone una ampliación significativa del área de distribución hacia zonas más occidentales de la provincia leonesa.

\section{Prunus insititia L.}

LEÓN: Rodrigatos de las Regueras, río Rodrigatos (Igüeña) (29TQH255332) 13-IV2002, borde de río, 1000 m, R. Martínez-Arias, LEB 79525; Carrizo de la Ribera (30TTN61) 15IV-1992, sebe en cultivo de lúpulo, E. Puente \& M.E. García, LEB 50380; San Juan de Paluezas (29TPH80) 30-VII-1988, encinar, A. Penas et al., LEB 31170.

Nuevas menciones para la provincia de este taxon que ya fue citado de Aleje (30TUN2646) y Ocejo de la Peña (30TUN2947), Alonso Redondo (2002); ambas localidades situadas mucho más al este que las nuestras.

\section{\# Rosa blondaeana Ripar ex Désegl.}

LEÓN: San Justo de Cabanillas (Noceda) 
(29TQH144299) 16-VI-2001, borde de camino, 830 m, R. Martínez-Arias, LEB 78125; Pantano de Bárcena (29TQH01) 17-V-1985, Penas et al., LEB 42068; Embalse de Bárcena (29TQH01) 17V-1985, Penas et al., LEB 42074; Peñarrubia (29TPH70) 19-V-1985, LEB 42063 y 18-V-1985, LEB 42064, Penas et al.; Llanos de Alba (30TTN84) 19-VII-1988, encinar, E. Puente \& C. Pérez Morales, LEB 38784, LEB 38783; Villafeliz de Babia VII-1973, robledal C. Romero (sub. R. nitidula).

Ya fue citada en dos ocasiones, Pola de Gordón (30TTN84) y La Robla (30TTN84) por Pérez Morales (1988) para la provincia con el sinónimo $R$. nitidula.

Nuestras poblaciones de San Justo de Cabanillas son las situadas más al noroeste de España.

\section{* Rosa elliptica Tausch}

LEÓN: Manzanal del Puerto (Villagatón) (29TQH283182) 23-VI-2001, borde de finca, 1195 m, R. Martínez-Arias, LEB 78125.

\section{\# Rubus caesius L.}

LEÓN: Salida a Folgoso de la Ribera, Km. 7 (29TQH197233) 16-VI-2001, camino forestal, 790 m, R. Martínez Arias, LEB 78109; Barrio de Nuestra Señora (30TUN03) 29-VI-1972, LEB 54400, Cofiñal (30TUN16) 30-VII-1986, sebe, LEB 53480 y Pantano de Luna (cruce de Aralla) (30TTN65) 9-VII-1986, borde de melojar, LEB 53472 , J. Andrés.

Varias veces citada esta especie en diferentes territorios eurosiberianos de la provincia.

Flora Iberica no la reconoce para León.

\section{* Rubus praecox Bertol.}

LEÓN: Barrio la Vega, Sorbera (Noceda) (29TQH117302) 1-VIII-2002, talud húmedo, 790 m, R. Martínez Arias, LEB 79207; Rodrigatos de las Regueras, arroyo de las Regueras (Igüeña) (29TQH256328) 31-VIII-2002, borde de arroyo, 975 m, R. Martínez-Arias, LEB 79186; Yebra (29TPG99) 14-VI-1985, sebe, I. Jiménez (sub. $R$. ulmifolius).

\section{Salvia sclarea L.}

LEÓN: San Pedro Mallo (Toreno)
(29TQH034364) 20-VII-2002, borde de carretera, 1080 m, R. Martínez-Arias, LEB 79212.

Segunda cita para la provincia de León de esta especie que fue mencionada por primera vez en Robledo de Losada 23-VIII-1983, M.J. Díez, SEV 90760, Díez et al. (1984). Supone además una ampliación significativa del areal de este taxon, que suele naturalizarse en herbazales nitrófilos próximos a poblaciones, hacia el norte de la provincia.

\section{Scilla ramburei Boiss.}

LEÓN: Matachana-Turienzo Castañero (Castropodame) (29TQH099186) 1-V-2002, melojar abierto, $745 \mathrm{~m}$, R. Martínez-Arias, LEB 79786.

Tercera mención de esta especie para la provincia de León que fue citada en el Puerto del Manzanal (29TQH24) por Laínz (1960) y en Vega de Espinareda, Burbia (29TPH8140), Silva Pando (1994).

+ Sedum andegavense (DC.) Desv.

LEÓN: Torre del Bierzo-La Granja de San Vicente (29TQH211206) 9-VI-2002, explanada ferroviaria con carbón, 790 m, R. Martínez-Arias, LEB 78329.

Segunda cita para León de este taxon que fue anteriormente citado en Santa Colomba de Somoza (29TQH20) 23-VI-1978, en pastizales de diente por Llamas García (1984). Supone una ampliación del areal hacia territorios más noroccidentales.

\section{* Spergularia purpurea (Pers.) G. Don. fil.}

LEÓN: Mirador de Boeza (Folgoso de la Ribera) (29TQH219299) 16-VI-2001, borde de carretera, 885 m, R. Martínez-Arias, LEB 78100.

\section{Veronica praecox All.}

LEÓN: Matachana-Turienzo Castañero (Castropodame) (29TQH099186) 1-V-2002, borde de melojar, $745 \mathrm{~m}$, R. Martínez-Arias, LEB 79552; San Andrés de los Puentes (Torre del Bierzo) (29TQH150182) 12-V-2002, prado, 750 m, R. Martínez-Arias, LEB 79630.

Ya fue citada para las localidades: Entre San Cristóbal de Valdueza y el Morredero (Ponferrada) (29TQG09), $1500 \mathrm{~m}$ y Corporales (Truchas) (29TQG08), $1200 \mathrm{~m}$; Aedo et al. 
(1994). Nuestras citas suponen una ampliación del área del taxon hacia el noroeste.

* Xanthium strumarium L. subsp. strumarium

LEÓN: Embalse de Bárcena, Santa Marina del Sil (Toreno) (29TQH039257) 6-IX-2001, llanura de inundación, $625 \mathrm{~m}$, R. Martínez-Arias, LEB 78743.

Primera mención de esta subespecie para la provincia leonesa. Este terófito que es característico de cunetas, baldíos y lugares ruderalizados, se diferencia de la subsp. italicum citada de la provincia, por tener unos frutos ovoides de menor tamaño que ésta $(<20 \mathrm{~mm})$, con los picos apicales rectos o muy poco curvados, que apenas sobresalen de los aguijones.

\section{BIBLIOGRAFÍA}

AEDO, C., JJ. ALDASORO, J.M. ARGÜELLES, J.L. DÍAZ ALONSO, A. DÍEZ RIOL, J.M. GONZÁLEZ. DEL VALLE, M. LAÍNZ, G. MORENO MORAL, J. PATALLO y O. SÁNCHEZ PEDRAJA -1994-. Contribuciones al conocimiento de la flora cantábrica, II. Fontqueria, 40: 67-100.

ALONSO REDONDO, R. -2002-. Valoración del estado de conservación de la vegetación y propuestas de ordenación y uso del territorio de la margen izquierda de la cuenca alta del río Esla. In Tesis Doctorales Publicadas en CD-ROM del 2000. Secretariado de Publicaciones y Medios Audiovisuales. Universidad de León.

CASTROVIEJO, S. et al. (eds.) -1986/2003-. Flora iberica. Plantas vasculares de la Península Ibérica e Islas Baleares, vol: IVIII, X y XIV. Real Jardín Botánico, CSIC. Madrid.

DÍEZ, M.J., J. PASTOR y I. FERNÁNDEZ. 1984-. Números cromosomáticos de plantas occidentales, 297-306. Anales Jard. Bot. Madrid 41(1): 191-194.

GARCÍA GONZÁlEZ., M.E., E. PUENTE GARCÍA, M.J. LÓPEZ PACHECO, L. HERRERO CEMBRANOS y A. PENAS MERINO -1987-. De plantis legionensibus. Notula X. Stud. Bot. Univ. Salamanca 6: 103108.
LAÍNZ, M. -1960-. Aportaciones al conocimiento de la flora cántabro-astur, IV. Bol. Inst. Estud. Astur. (Supl. Ci.) 1: 3-42.

LAÍNZ, M. -1964-. Aportaciones al conocimiento de la flora cántabro-astur, VIII. Bol. Inst. Estud. Astur. (Supl. Ci.) 10: 173-218.

LlamAs, F., C. ACEDO, R. ALONSO, C. LENCE, S. DEL RÍO y A. FERNÁNDEZ 2003-. Flora leonesa amenazada. Acta Bot. Barc, 49: 53-66.

LLAMAS GARCÍA, F. -1984-. Flora $y$ vegetación de la Maragatería (León). Institución "Fray Bernardino de Sahagún». Excma. Diputación Provincial de León. Consejo Superior de Investigaciones Científicas (CECEL).

LÓPEZ PACHECO, M.J. -1988-. Flora y vegetación de las cuencas alta y media del río Curueño (León). Diputación Provincial de León. Institución Fray Bernardino de Sahagún. León.

NIETO FELINER, G. -1985-. Estudio crítico de la flora orófila del Suroeste de León: Montes Aquilianos, Sierra del Teleno y Sierra de la Cabrera. Ruizia 2: 5-237.

PAU, C. -1893-. Plantas españolas recogidas el otoño pasado por mi distinguido amigo y colega Sr. A.E. Lomax, de Liverpool, según muestras enviadas por él mismo. Actas Soc. Esp. Hist. Nat, 22(2): 77-89.

PAZ CANURIA, E., M.E. GARCÍA GONZÁLEZ., E. ALONSO HERRERO y A. PENAS MERINO -1999-. Referencias corológicas de plantas vasculares en León y localidades próximas de las provincias de Valladolid y Zamora (España). Acta Bot. Malacitana 24: 198-204.

PENAS MERINO, A. -1984-. Nuevos taxónes para la flora leonesa. Lagascalia 13(1): 3-16.

PÉREZ GARCÍA, M.A. -1983-. Flora y vegetación de la comarca de la Omaña. Tesis Doctoral. Facultad de Farmacia de Santiago de Compostela.

PÉREZ MORALES, C. -1988-. Flora y vegetación de la cuenca alta del río Bernesga. Diputación Provincial de León. Institución Fray Bernardino de Sahagún. León.

PONGA PÉREZ, M.T. -1984-. Estudio de la flora $y$ vegetación de las gleras calizas cantábricas. Memoria de Licenciatura. 
Departamento de Botánica. Facultad de Ciencias Biológicas. Universidad de León.

PUENTE GARCÍA, E. -1988-. Flora y vegetación de la cuenca alta del río Sil (León). Diputación Provincial de León. Institución Fray Bernardino de Sahagún. León.

ROMERO ZARCO, C. -1990-. Las avenas del grupo barbata en la Península Ibérica e Islas Baleares. Lagascalia 16(2): 243-268.

SILVA PANDO, F.J. -1994-. Flora y series de vegetación de la Sierra de Ancares. Fontqueria 40: 233-388.

VALCÁRCEL, V, H.A. McALLister, A. RUTHERFORD y R.R. MILL -2003-. Hedera
L. In CASTROVIEJO, S. et al. (eds.). Flora iberica. Plantas vasculares de la Península Ibérica e Islas Baleares, vol. X. Real Jardín Botánico, CSIC. Madrid.

Aceptado para su publicación en junio de 2004

Dirección de la autoras. Dpto. Biología Vegetal (Botánica). Campus de Vegazana Universidad de León. 24071 León (Spain). E-mail: dbvrma@,unileon.es

\title{
140. ACERCA DE SIDERITIS BORGIAE ANDRÉS SUBSP. BORGIAE (LAMIACEAE)
}

\author{
Marta Eva GARCÍA GONZÁLEZ, Raquel ALONSO REDONDO \\ y Ruth MARTÍNEZ ARIAS.
}

About Sideritis borgiae Andrés subsp. borgiae (Lamiaceae).

Palabras clave. Sideritis borgiae, Lamiaceae, corología, España.

Key words. Sideritis borgiae, Lamiaceae, chorology, Spain.

Palencia: Camporredondo de Alba, 30TUN64, $1345 \mathrm{~m}$, en brezal del Pterosparto lasianthi-Ericetum aragonensis hypericetosum burseri, 8-VII-2003, M.E. García González, R. Alonso Redondo \& R. Martínez Arias, LEB 82399 (Figura 1).
Este endemismo de areal restringido, ha sido recientemente incluido en la Lista Roja de la Flora Vascular Española [VV.AA., Conserv. Veg. 6 (extra): 11-38, 2000] en la categoría DD (datos insuficientes), tanto por su distribución aún poco conocida, como por

Este trabajo ha sido realizado en el marco del Proyecto de Investigación "Cartografía detallada de hábitats del Anexo I de la directiva 92/43/CEE a escala 1:10.000 en espacios incluidos en la Red Natura 2000", contrato suscrito con la Universidad de Salamanca para realizar dicho proyecto en 15.000 Has del Parque Natural de Fuentes Carrionas y Fuente Cobre- Montaña Palentina. 